\title{
Sur les noms de paroisses au Québec, des origines à 1925
}

\section{Pierre Savard}

Volume 41, 1974

URI : https://id.erudit.org/iderudit/1007244ar

DOI : https://doi.org/10.7202/1007244ar

Aller au sommaire du numéro

Éditeur(s)

Les Éditions Historia Ecclesiæ Catholicæ Canadensis Inc.

ISSN

0318-6172 (imprimé)

1927-7067 (numérique)

Découvrir la revue

Citer cet article

Savard, P. (1974). Sur les noms de paroisses au Québec, des origines à 1925.

Sessions d'étude - Société canadienne d'histoire de l'Église catholique, 41,

105-113. https://doi.org/10.7202/1007244ar

Tous droits réservés @ Ces Éditions Historia Ecclesiæ Catholicæ Canadensis Inc., 1975
Ce document est protégé par la loi sur le droit d'auteur. L’utilisation des services d'Érudit (y compris la reproduction) est assujettie à sa politique d'utilisation que vous pouvez consulter en ligne.

https://apropos.erudit.org/fr/usagers/politique-dutilisation/ 


\section{Sur les noms de paroisses au Québec, des origines à 1925 *}

\section{Introduction}

Par l'abondance du matériau, le Québec offre le cas le plus poussé au monde où le nom d'un saint avec son préfixe s'applique à des noms de paroisses tant civiles que religieuses ${ }^{1}$. À l'abondance du matériau s'ajoute un autre motif d'intérêt, soit son étalement sur trois siècles à un moment où, en Europe, on crée relativement peu de toponymes religieux. Celui qui veut suivre l'évolution du sentiment religieux catholique à travers l'hagionymie doit passer les mers et observer les créations de la vallée du Saint-Laurent qui prend comme le relais de la vieille Europe dont les grands traits de l'hagionymie sont déjà fixés à la fin du haut moyen âge ${ }^{2}$.

Cet ensemble de données a peu attiré l'attention jusqu'ici. Nos spécialistes de la typonymie ont le plus souvent été mus par des soucis normatifs plus que par la recherche historique comme telle (purger la carte, redresser les erreurs, lutter contre les corruptions onomastiques, réhabiliter les toponymes indigènes, etc. $)^{3}$. Les auteurs

* Communication présentée au congrès annuel de 1974 de la Société Canadienne d'Histoire de l'Eglise Catholique.

1 Le phénomène est déjà cité au siècle dernier, par exemple dans le Dictionnaire géographique et administratif de JoANNE, p. 4024. La France suit loin en arrière avec une commune sur huit environ portant le nom d'un saint avec son préfixe.

2 Bel exemple rappelé dans JoANNE cité que celui de Sainte-Marie, assez peu fréquent du fait que le culte marial ne se répand qu'à partir des croisades avec saint Bernard et les cisterciens alors que les lieux sont déjà nommés.

3 La choronymie qui englobe un domaine plus large que la toponymie est l'objet des travaux d'un groupe dynamique de chercheurs de Laval autour de Henri Dorion et de Fernand GrENIER (Groupe d'étude de choronymie et de terminologie géographique). Voir, à titre d'exemple de leurs travaux, Christian BonNely, Jean-Marc Nicole et Judith RoY, «Le processus de formation et d'évolution des hagionymes. Exemples tirés de trois inventaires choronymiques dans l'Est du Québec », dans Cahiers de géographie de Québec, vol. 16, $\mathrm{n}^{\circ} 37$ (avril 1972), pp. 99-112. Jean PoIRIER, secrétaire de la Commission de géographie du Québec au Ministère des Terres et Forêts, se penche depuis une quinzaine d'années sur la toponymie. Voir son article récent : " $\mathbf{L a}$ toponymie québécoise : une image du milieu humain où s'attachent des vestiges du passé », dans Forces Hydro-Québec, numéro 14/1971 (avec une bibliographie sommaire). Sur la fonction normative de la Commission de Géographie de Québec, voir l'exposé de Jean POIRIER dans l'Annuaire du Québec 1973 , pp. $544-545$. 
de la littérature ont su parfois puiser avec bonheur dans ce stock si riche de symboles ${ }^{4}$.

Nous nous penchons ici sur les noms de paroisses du début du XVII ${ }^{\mathrm{e}}$ siècle jusqu'à 1925 pour y chercher des enseignements sur la culture religieuse et profane des hommes d'ici. Nous tirons notre documentation de la compilation de Hormidas Magnan, Dictionnaire historique et géographique des paroisses, missions et municipalités de la province de Québec (...) (Arthabaska, 1925). Ce livre de 738 pages renferme les noms de 1130 paroisses ayant un curé résident et 138 dessertes et missions. Pour rédiger cette «histoire abrégée de toutes les paroisses et missions de la province de Québec ", l'auteur a consulté plus de cent histoires de paroisses ${ }^{5}$. La méthode que nous suivons est empirique. Un dépouillement de la littérature de ce type en France durant les vingt dernières années nous a peu apporté du fait que les chercheurs français se préoccupent avant tout des rapports entre toponymie et archéologie, en général du haut moyen âge.

\section{Un univers religieux de tous les temps}

Le Christ sous divers attributs occupe une place de choix du début à la fin de la période étudiée (de l'Enfant-Jésus de la Pointe-

4 Contentons-nous de citer ici une belle page de Mater Europa (Paris, 1968) de Jean-Éthier BLaIs qui montre les ressources inépuisables d'un tel monde : "Cette ville (Montréal) a une physionomie tragique et ce n'est pas en vain que la rue qui la rejette de part et d'autre, du nord au sud, en deux tronçons, a emprunté son nom à un saint cuit et recuit sur le gril. SaintLaurent est tout aussi présent à Montréal qu'à Escurial. Montréal s'étend en effet, entre le fleuve Saint-Laurent... » (pp. 78-79). Claude RACINE, dans L'anticléricalisme dans le roman québécois, 1940-1965; (Montréal, 1972), rélève les traits du romancier Bertrand Vac contre le caractère «pieusard》 de notre toponymie (p. 92). L'hagionymie québécoise a fourni un aliment riche à l'ironie anticléricale.

5 « La table chronologique des paroisses et municipalités de la province de Québec tenant registre de 1621 à 1871 », publiée par Cyprien TANGUAY dans le premier tome de son Dictionnaire (...), pp. 601-605, renseigne sur la distribution des noms dans le temps. De 1660 à 1830 on crée une dizaine de paroisses par an. Le mouvement s'accélère après pour atteindre le sommet de 117 érections canoniques dans la décennie de 1850, période de colonisation intense. Lourd reste le poids du XIXe siècle dans notre toponymie comme dans notre histoire religieuse. 
aux-Trembles $/ M / 1678$ à Saint-Rédempteur $/ Q / 1919)^{6}$. Après 1867, c'est surtout sous les traits du Sacré-Cour qu'on connaît le Christ : 15 érections canoniques sans compter les noms reliés au culte comme Marie-Alacoque, Notre-Dame-du-Sacré-Cour et le Précieux-Sang (2 paroisses après 1900). Le culte eucharistique apparaît explicitement après 1885 avec trois Saint-Sacrement et se continue dans deux Saint-Pascal-Baylon (1910 et 1923). Les autres attributs notables sont l'Enfant-Jésus (4 paroisses) à relier à la Sainte-Famille (voir plus bas), Saint-Sauveur et Saint-Rédempteur (4 noms après 1880). La Sainte Trinité apparaît trois fois, l'Ascension, deux fois.

Le nombre des dédicaces à la Vierge Marie, soit quelque 80 , représente le double de celles au Christ. Ici encore, il faut distinguer par attributs. L'Immaculée-Conception et Notre-Dame de Lourdes (après 1878) totalisent 16 entrées. Viennent ensuite la Visitation (8), Notre-Dame du Rosaire et Saint-Cour de Marie ( 7 chacune), NotreDame du Bon Conseil et l'Assomption (6 chacune), Notre-Dame-deBon-Secours (4) et Notre-Dame du Perpétuel Secours (3), NotreDame-des-Victoires (4), Notre-Dame-de-la-Paix et Notre-Dame-desNeiges ( 3 chacune). Dans le groupe des lieux de pèlerinages associés au culte marial ce sont Lourdes (6), le Mont-Carmel (6) et Lorette (3) qui dominent.

Avec 35 érections canoniques, le culte de saint Joseph apparaît fort répandu. Le premier patron des Canadiens français figure dès 1694( Saint-Joseph-de-la-Pointe-Lévis /Q). En 1925 encore, l'archevêque de Québec met une nouvelle paroisse de la ville sous ce patronage pour commémorer le troisième centenaire du saint comme patron du Canada.

Six dédicaces à la Sainte-Famille bien distribuées dans le temps et dans l'espace (Q/1684 à T.-R./1918) attestent une dévotion durable.

Avec vingt-quatre dédicaces, sainte Anne occupe une place de choix, depuis 1678 (Sainte-Anne-de-la-Pocatière/Q) jusqu'à 1919 (Sainte-Anne-du-Lac/M.-L.). Ces paroisses sont souvent des lieux de pèlerinage. $\mathrm{La}$ dédicace entraîne dans 7 cas une dédicace voisine

6 Nous donnons le nom religieux suivi de la date d'érection et du nom du diocèse en 1922 suivant les abréviations: C: Chicoutimi; M: Montréal; M.-L.: Mont-Laurier; N: Nicolet; Q: Québec; R: Rimouski; Sh.: Sherbrooke; St-H.: Saint-Hyacinthe; T.-R.: Trois-Rivières. 
à Saint-Joachim : il s'agit le plus souvent de détachements de la paroisse de Sainte-Anne.

Certains apôtres le disputent à sainte Anne en popularité. Pierre apôtre donne son nom à 19 paroisses des 24 qui portent le nom (depuis Saint-Pierre-de-Sorel/1670/St.-H.). Souvent dans le voisinage du premier, on trouve des Paul apôtres (17 sur 18 SaintPaul) (depuis Saint-Pierre et Saint-Paul/1714/Q, devenu SaintLaurent dans l'île d'Orléans). L'apôtre Jean a donné son nom à 12 paroisses, Jacques le Majeur (9), André (9), Thomas (7), Philippe (7), Mathias (4), Jacques le Mineur (2), Barnabé (3), Barthélemi (2), Simon (4) associé une fois à Jude.

Du peloton des saints ordinaires se détache nettement SaintJean-Baptiste avec 17 dédicaces depuis 1714 (Q).

Le bataillon serré des Anges occupe beaucoup de place. SaintMichel est le plus répandu (16 dédicaces) suivi de Gabriel (8) et de Raphaël (6). Les Saints-Anges, L'Ange-Gardien et Les AngesGardiens totalisent 8 paroisses.

Pour introduire une certaine cohérence et sans trop forcer l'explication, tentons de regrouper les saints qui restent suivant des affinités.

Le cycle des jésuites, missionnaires installés dans la colonie dès le $\mathrm{XVII}{ }^{\mathrm{e}}$ siècle et qui reviennent en force au milieu du XIX, apparaît un des plus riches avec 16 François-Xavier, 7 Ignace et 5 Louis-de-Gonzague.

Le cycle récollet-franciscain-capucin, avec environ 19 SaintAntoine-de-Padoue (pas toujours facile à distinguer de Antoine Abbé), 8 Saint-François-d'Assise et 3 Saint-Bernardin, se révèle également riche.

On peut regrouper sous la rubrique du cycle «français 》 des saints de la France associés à la grandeur à la fois politique et religieuse de ce pays : Saint-Louis (23 dédicaces), Sainte-Jeanned'Arc (8, toutes entre 1920 date de la canonisation et 1922), Rémi (6), Clotilde (5), sans compter Bernard (5) et Martin-de-Tours (2).

L'univers des saints irlandais est bien représenté avec en tête Patrice (de-la-Rivière-du-Loup/1833/Q), Brigitte (6), Colomban (3) et Malachie (3).

Un certain nombre de saints « catacombaires » rappellent les persécutions des débuts du christianisme comme Cécile (10), Agnès (4) et Philomène (4) : ils apparaissent au $\mathrm{XIX}^{\mathrm{e}}$ siècle à la suite 
des révélations de la Rome souterraine et symbolisent l'Église et les chrétiens assiégés dans un monde moderne hostile.

Il est plus difficile de regrouper des saints relativement répandus comme Saint-Charles (14), Saint-Édouard (13), Saint-Étienne (10), Saint-Georges (10), Saint-Alphonse (8), Sainte-Rome-de-Lima (8, depuis 1846), Saint-Augustin (7), Saint-Roch (7), Saint-Dominique (6) et Sainte-Elisabeth (6).

\section{La question des origines : une simple question de patronymie?}

On ne peut manquer de se poser la question des origines. La réponse la plus courante est de renvoyer au hasard du calendrier (la fête du jour) ou mieux encore, au nom de baptême du premier colon, du premier curé ou d'un proche du seigneur. L'analyse mérite toutefois d'être poussée plus loin.

Les saints choisis doivent répondre à un certain nombre de critères implicites. Ils doivent d'abord être de quelque importance dans l'Église universelle. Le martyrologe romain compte quelque 4,500 noms. Or, le nombre des noms qu'on retrouve au Québec reste bien réduit : il $\mathrm{y}$ a donc eu sévère sélection pour des raisons théologiques ou canoniques. Signalons en passant le cas intéressant des rejets explicites. Rejets rares et surtout d'ordre euphonique tel celui de Saint-Abdon, devenu Saint-Luc (1919/Q) ou de SaintRaymond-Nonnat devenu Saint-Grégoire-le-Grand (1836/St-H.). Le hasard du calendrier ne suffit pas à tout expliquer même si on le retrouve nommément dans une douzaine de cas. Ni ne peut suffire le fait que le saint ou la sainte vienne d'être canonisée (ex.: SainteGermaine/1869/Q) ou Saint-Vianney/1921/R). Ajoutons qu'une mécanique certaine entraîne des associations (24 relevées) dont les plus fréquentes sont : Cyrille et Méthode (2); Monique et Augustin (2); Luc ou Timothée et Tite (2); Marthe ou Marie-Madeleine et Lazare (2). La dévotion particulière de l'évêque ${ }^{7}$ ou des habitants $\mathrm{du}$ lieu explique un bien petit nombre de choix ${ }^{8}$.

7 Par exemple, Mgr Bégin de Québec, qui a étudié à Rome, aime rappeler les souvenirs de la Ville Éternelle (Sainte-Sabine, 1906/Q; SaintBenoît-Labre, 1893/Q).

8 Par exemple, Sainte-Geneviève-de-Batiscan, 1833/T.-R.; Saint-Léonard-de-Port-Maurice, $1885 / \mathrm{M}$ (le saint a été canonisé en 1867). On ne saurait parler ici de religion populaire qu'avec une grande prudence. Les noms sont venus presque dans tous les cas d'en-haut (évêque ou curé). La dévotion du peuple n'a pu que suivre quand elle a suivi, ce qui reste à démontrer. 
On aurait tort de tout réduire au problème des origines. Une fois le nom donné, les paroissiens sont appelés à vivre avec un saint qu'ils n'ont peut-être pas désiré mais qui va entrer dans leur univers familier : un tableau le représentant occupe souvent une place de choix au-dessus du maitre-autel et le curé célèbre à l'occasion ses mérites. Tous ces saints littéralement importés de l'Ancien Monde font vivre les fidèles dans un ailleurs et un hier pleins de merveilleux décrits dans La Légende dorée.

Ce n'est pas ici qu'il faut chercher un riche matériau sur la vie politique du temps (sauf dans des cas limites comme Sainte Victoire (en l'honneur de Victoria, N/1863), ni sur la vie socioéconomique. Les moyens de production apparaissent bien peu représentés avec 5 Isidore et un Agricol. Même si on rattache quelque peu abusivement Sainte-Anne aux marins du Saint-Laurent. Et rien ne nous permet encore d'associer, durant cette période, saint Joseph à la vie ouvrière.

Il semble par contre plus révélateur de relever quelques traits de l'anthroponymie liés à la place du clergé et au rôle des élites laïques. De l'ensemble des noms explicitement patronymiques, près de la moitié rappellent le prénom du premier curé ou du premier desservant ( 3 cas rappellent le prénom du curé dont la paroisse a été détachée). Un nom sur six vient du choix explicite de l'évêque ou du seigneur ecclésiastique avant 1854 : prénom de l'évêque dans la moitié des cas ou encore plus rarement, nom de sa mère, de sa sœur, voire de sa nièce. Les laïcs, eux, se partagent un bon tiers des noms de paroisses. Avant l'abolition du régime seigneurial, le nom vient le plus souvent du seigneur, de sa femme ou de sa fille. Après 1854 c'est aussi souvent le nom du premier colon que celui d'un notable bienfaiteur de la paroisse, notaire ou riche marchand; les seigneurs ont disparu, faisant place aux nouvelles élites.

Le pays d'ici apparaît timidement dans des Notre-Dame-desNeiges (3) qui rappellent aussi, bien sûr, le miracle sur l'emplacement de la basilique romaine de Sainte-Marie-Majeure.

\section{L'évolution du sentiment religieux}

Ces centaines de noms de saints ont été donnés dans une conjoncture temporelle particulière qui leur fait refléter des états successifs de sensibilité religieuse. 
Aux XVII et XVIII e siècles, "grands siècles des âmes », les choix reflètent sans doute surtout la spiritualité d'un $\mathbf{M}^{\mathrm{gr}}$ Laval ou d'un $M^{\text {gr }}$ de St-Valier elles-mêmes en accord avec leur temps. Le petit nombre des choix reste peu instructif : comme dans tous les diocèses naissants on puise dans un stock de saints classiques, à commencer par les apôtres. Soulignons que la conquête de la Nouvelle-France ne marque ici aucune rupture.

La première moitié du $\mathrm{XIX}^{\mathrm{e}}$ siècle, période de créations nombreuses, voit une des vagues caractérisées. Les noms irlandais font leur apparition dans les années 1830 avec l'arrivée des immigrants de l' "Île des Saints " suivis de près par les saints «catacombaires » comme sainte Philomène au succès durable ${ }^{9}$. Le prosélytisme protestant entraîne dans la région de Montréal une offensive de dédicace à des pères de l'Église qui ont lutté contre l'hérésie (Saint-Hermas/ 1834).

La seconde moitié du siècle voit le retour ou l'arrivée de congrégations religieuses qui vont laisser leur empreinte sur la carte de l'hagionymie. Nous avons dit l'importance des cycles jésuitiques (retour dans les années 1840) et franciscains (retour dans les années 1890). Très important, quoique plus difficilement mesurable, est le rôle des oblats qui répandent le culte marial après 1840 . Il faut sans doute associer aux rédemptoristes les Saint-Alphonse-de-Liguori. Après 1900 surtout, les Eudistes multiplient les dédicaces au Coeur-de-Marie.

La dévotion au pape qui se répand sous Pie IX (1846-1878) apparaît dans quelques dédicaces à Saint Pie (et à Saint-Léon après 1878).

Dans le dernier quart du XIX ${ }^{e}$ siècle et le premier quart de celui-ci, les saints liés à la grandeur de la France se multiplient. Floraison liée sans doute aux vagues d'émigration de religieux sur nos bords, suite aux lois Ferry et à la Séparation de 1905.

La guerre de 1914 vaut quelques noms de paroisses (NotreDame-de-la-Paix/Q) et les Néo-Canadiens révèlent timidement leur existence après 1900 (Notre-Dame de la Difesa/M). Saint-Christophe patron des automobilistes est donné à une paroisse du Nord de Montréal en 1915 (M) alors que les véhicules motorisés commencent à se répandre sur les routes.

9 Auxquels on peut ajouter plusieurs Zotique à partir de 1849 quand Mgr Bourget apporte au pays les reliques du saint. 


\section{Conclusion}

L'univers religieux des noms de paroisse au Québec du XVII siècle à 1925 frappe par son abondance et sa richesse. Tout au long des trois siècles il fait une place de choix aux grands cultes de l'Église : le Christ et la Vierge sous divers attributs; les apôtres Pierre, Paul et Jean et les anges y occupent une grande place; les grands saints de l'Église universelle comme Joseph, François-Xavier, Jean-Baptiste, Cécile, Étienne, Georges, Alphonse, Augustin, François d'Assise, Ignace et Roch.

Les origines de cette chrétienté qui fut longtemps un rameau de l'Église gallicane et qui reçut pendant toute la période des renforts cléricaux de la «fille aimée de l'Église », est attestée par l'abondance des Louis de France et des Jeanne d'Arc (au XX ${ }^{\mathrm{e}} \mathbf{s}$.). Il faut aussi expliquer par les origines françaises l'étonnante floraison de la dévotion à sainte Anne, dévotion bien enracinée et ravivée dans le dernier tiers du $\mathrm{XIX}^{\mathrm{e}}$ siècle.

L'arrivée de catholiques d'autres origines ethniques se révèle dans la toponymie religieuse avec les hagionymes irlandais abondants après 1830 .

Des congrégations religieuses qui ont surtout marqué la toponymie, il faut citer les jésuites durant presque toute la période et les franciscains; plus difficile à évaluer mais considérable est le rôle des oblats et des eudistes dans le choix des noms mariaux.

Si on serre de près la chronologie, on voit apparaitre dans la vallée du Saint-Laurent une chrétienté qui reflète fidèlement ses métropoles spirituelles, la France surtout durant deux siècles puis Rome au XIX ${ }^{\mathrm{e}}$ siècle et au $\mathrm{XX}^{\mathrm{e}}$ siècle. Aux saints classiques succèdent après 1800 les saints «catacombaires 》 remis en vogue par les Papes auxquels viennent s'ajouter après 1870 surtout les saints «français » revivifiés par les catholiques de la Troisième République. Le $\mathrm{XX}^{\mathrm{e}}$ siècle voit déferler sur les bords du Saint-Laurent les vagues de dévotion qui secouent l'Église universelle (Sacré-Cœur, Eucharistie, curé d'Ars).

Bien entendu tout ne saurait se réduire à une question de source. Le catholicisme québécois ne saurait se réduire à un reflet ou un appendice d'un mouvement général. Des dévotions solidement installées ici et revivifiées au moment opportun ont pesé plus lourdement que des cultes éphémères qui n'ont laissé qu'un nom de 
paroisse quand culte il y a eu. Sainte Anne et saint Jean-Baptiste présents tout au long de notre enquête méritent plus d'attention que cinquante saints qui dorment au cimetière de l'hagionymie.

Cette étude appelle des prolongements. Dans le temps d'abord. Même si nous ne disposons pas d'un instrument équivalent au Magnan après 1925, l'étude du dernier demi-siècle, riche en mutations sociales et religieuses, peut se faire à l'aide du Canada ecclésiastique (annuel) et des monographies paroissiales aujourd'hui bien inventoriées. Dans l'espace, il faudrait étendre l'enquête aux francophones de la diaspora tant canadienne qu'états-unienne. La profonde unité culturelle de ces groupes dispersés en même temps que leur spécificité régionale en sortiraient éclairées. Enfin, des comparaisons avec d'autres chrétientés de la même époque révéleraient l'originalité du catholicisme d'ici tout en aidant à comprendre son rôle dans la culture qui a poussé sur les bords du Saint-Laurent.

Pierre SAVARD,

Professeur agrégé, Département d'Histoire, Université d'Ottawa. 